\title{
Three Cases of Acquired Simulated Brown Syndrome after Blowout Fracture Operations
}

\author{
So Young Ji, Jae Hong Yoo, Won Ha, Ji Won Lee, Wan Suk Yang \\ Department of Plastic and Reconstructive Surgery, Dong Kang General Hospital, Ulsan, Korea
}

Brown syndrome is known as limited elevation of the affected eye during adduction. It is caused by a disorder of the superior oblique tendon, which makes it difficult for the eyeball to look upward, especially during adduction. It is classified into congenital true sheath Brown syndrome and acquired simulated Brown syndrome. Acquired simulated Brown syndrome can be caused by trauma, infection, or inflammatory conditions. The surgical restoration of blowout fractures can also lead to limitations of ocular motility, including Brown syndrome. We report on three patients with acquired simulated Brown syndrome, who complained of diplopia and limitation of ocular motility after operations to treat blowout fractures.

Keywords Brown-Sequard syndrome / Ocular motility disorder

\author{
Correspondence: Wan Suk Yang \\ Department of Plastic and \\ Reconstructive Surgery, Dong Kang \\ General Hospital, 239 Taehwa-ro, \\ Jung-gu, Ulsan 681-711, Korea \\ Tel: +82-52-241-1738 \\ Fax: +82-52-241-1738 \\ E-mail:artps@naver.com
}

No potential conflict of interest relevant to this article was reported.

Received: 18 Aug 2014 • Revised: 6 Dec 2014 • Accepted: 9 Dec 2014

pISSN: 2234-6163 • elSSN: 2234-6171 • http://dx.doi.org/10.5999/aps.2015.42.3.346• Arch Plast Surg 2015;42:346-350

\section{INTRODUCTION}

Brown syndrome is a congenital or acquired ocular motility disorder, characterized by limited elevation in the adduction of the affected eye [1]. It is classified into true sheath and simulated Brown syndrome, based on whether a congenitally short anterior sheath of the superior oblique tendon is the only cause of the syndrome [2].

Infection, inflammatory disease, and trauma can cause acquired simulated Brown syndrome [3]. It can also be caused by surgical trauma, glaucoma drainage implantation, ethmoidal sinus surgery, and tucking of the superior oblique tendon [4]. In addition, the surgical repair of a blowout fracture can injure the trochlea and superior oblique muscle-tendon complex, which in turn can result in acquired simulated Brown syndrome [5].

We report on three cases of acquired simulated Brown syndrome that occurred after blowout fracture operations and then discuss strategies to prevent and treat such cases.

\section{CASES}

\section{Case 1}

A 37-year-old man visited the emergency room with a left periorbital injury caused by a traffic accident. An initial examination showed no abnormalities in visual acuity, intraocular pressure, or the fundus. The preoperative diagnosis was a blowout fracture involving the medial wall of the left orbit (Fig. 1). Surgical repair of the fracture through insertion of a Medpor (Stryker, Kalamazoo, MI, USA) implant was performed six days after the injury. After the operation, the patient complained of newly developed diplopia. The elevation of the left eye was conspicuously limited during adduction (Fig. 2). A forced duction test of the left eye showed restricted elevation during adduction. A postoperative computed tomography (CT) scan of the orbit showed that the left superior oblique muscle was trapped between the inserted Medpor fragment and the border of the fracture (Fig. 3). Surgical re-exploration was performed and the Medpor im- 
plant was removed (Fig. 4). Fortunately, reduction using a bone hook allowed the defect to be almost completely covered with the depressed orbital wall. No artificial bone was inserted. After surgery, a forced duction test showed no restriction of ocular motility. However, the diplopia remained for two months postoperatively and was corrected with prism glasses. At an eightmonth follow-up visit, the patient's diplopia was alleviated and his ocular motility was significantly improved (Fig. 5).

\section{Case 2}

A 52-year-old man came to the hospital with left periorbital blunt

Fig. 1. A preoperative computed tomography scan, coronal view

A computed tomography scan demonstrates left medial wall fractures (case 1).

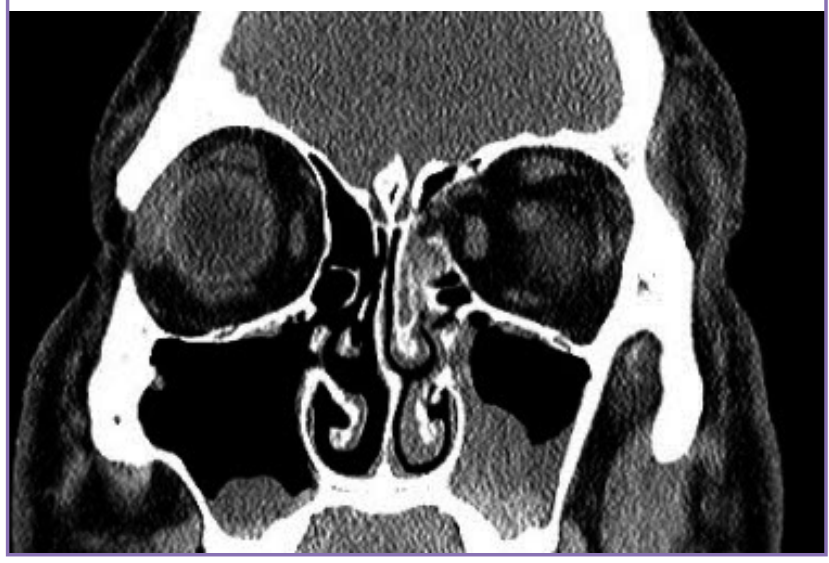

trauma caused by a machine tool. He complained of periorbital ecchymosis and subconjunctival hemorrhage without diplopia and ocular motility limitation. An orbital CT scan revealed a left medial blowout fracture (Fig. 6). Surgical repair with the insertion of a Medpor implant via a transcaruncular approach was performed five days after the injury. Immediately after the operation, he complained of diplopia and limited ocular motility. A forced duction test was positive, demonstrating restriction of elevation during adduction of the left eye. A postoperative $\mathrm{CT}$ scan showed entrapment of the superior oblique muscle between the

Fig. 3. A postoperative computed tomography scan

A reconstructed blowout fracture involving the left medial orbital wall is shown. The left medial rectus muscle is swollen. Red arrow: The superior oblique muscle is trapped between the Medpor implant and the superior orbital fracture margin (case 1).

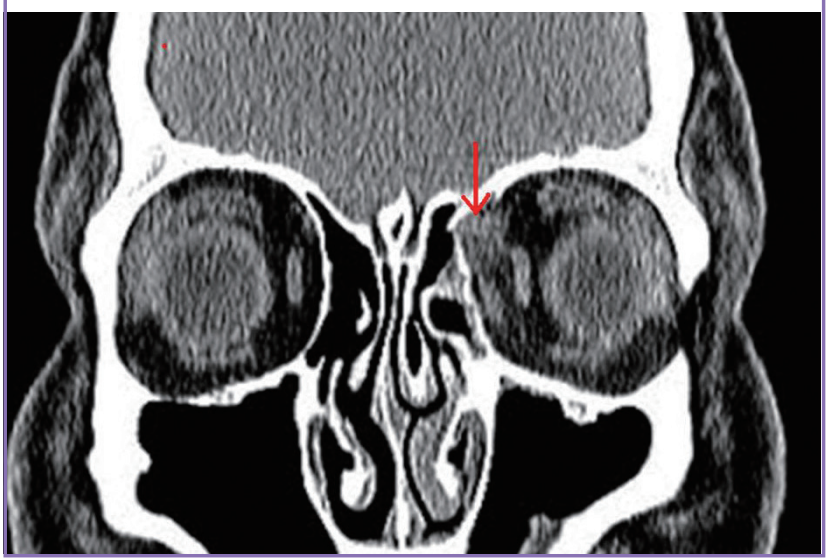

Fig. 2. A postoperative photograph of blowout fracture

A postoperative photograph of a 37-old-male patient following the surgical repair of a left medial blowout fracture shows limited elevation during the adduction of his left eye (case 1).
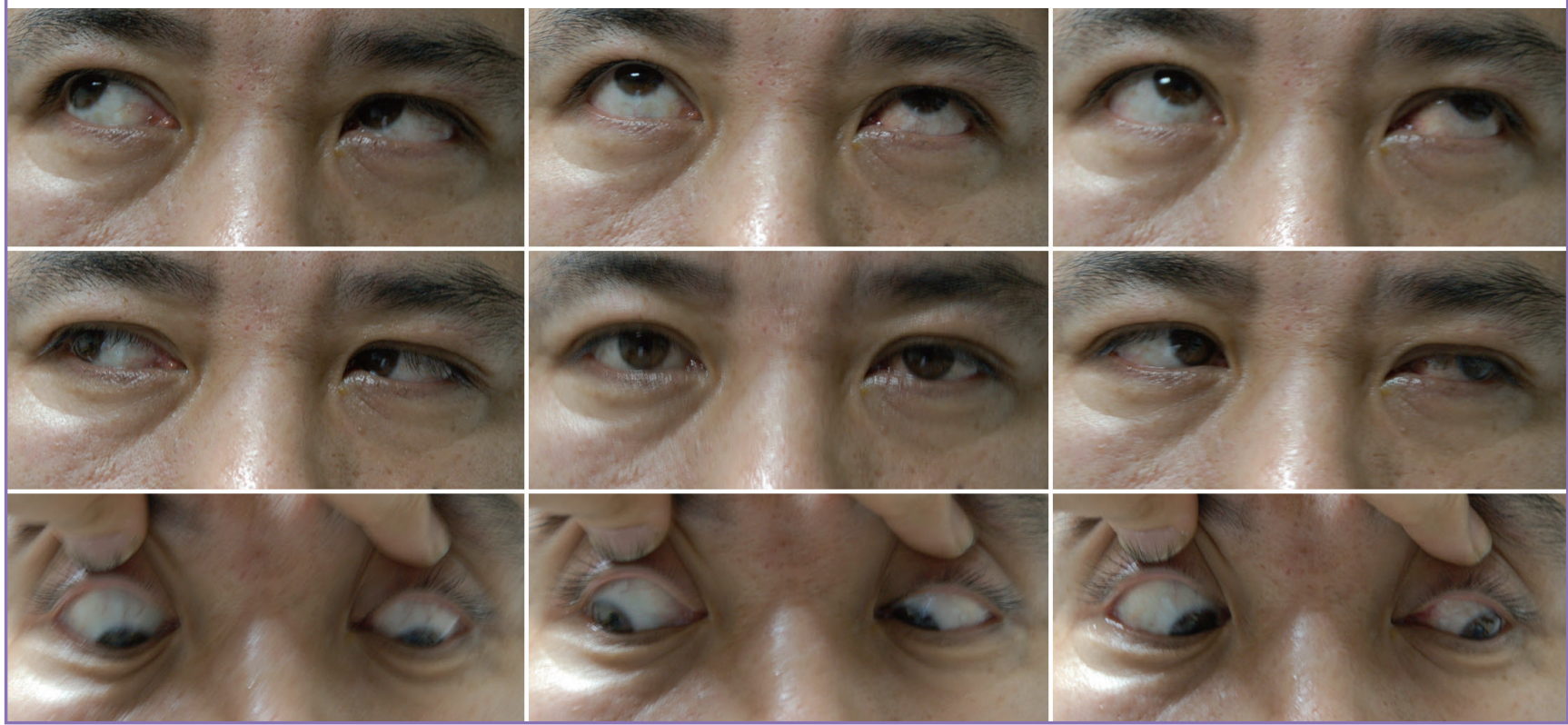

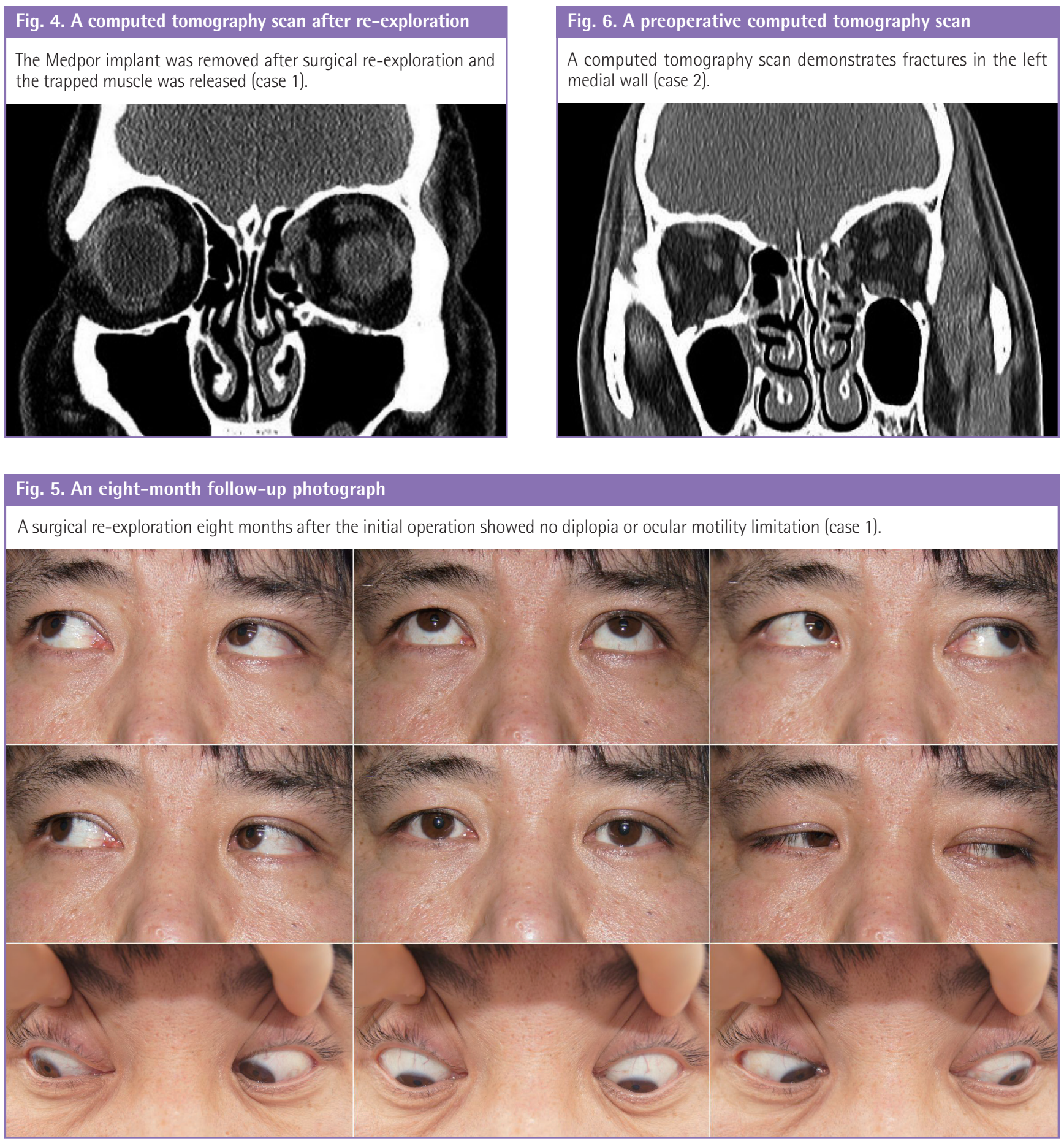

Medpor implant and the orbital wall fracture border (Fig. 7). Surgical re-exploration was promptly performed and the entrapped muscle was released by removing the Medpor implant. A newly designed Medpor implant was reinserted into the depressed orbital wall (Fig. 8). After three month, the diplopia and ocular motility limitation were completely resolved.

\section{Case 3}

A 52-year-old man was transferred to our department for the surgical repair of a right medial blowout fracture caused by a violent assault. At first, he complained of diplopia in all directions of gaze. An examination showed limited movement in the right eye during abduction. Traumatic hyphema and vitreous hemorrhage were observed. After the hyphema and vitreous hemorrhage were completely stabilized, an operation was performed to correct the blowout fracture. The herniated orbital fat and muscle were restored and a Medpor implant was inserted into the defect of the orbital wall using a transcaruncular approach. 


\section{Fig. 7. A postoperative computed tomography scan}

A reconstructed blowout fracture involving the left medial orbital wall is shown. Red arrow: A postoperative computed tomography scan showed entrapment of the superior oblique muscle between the Medpor implant and the orbital wall fracture border (case 2).

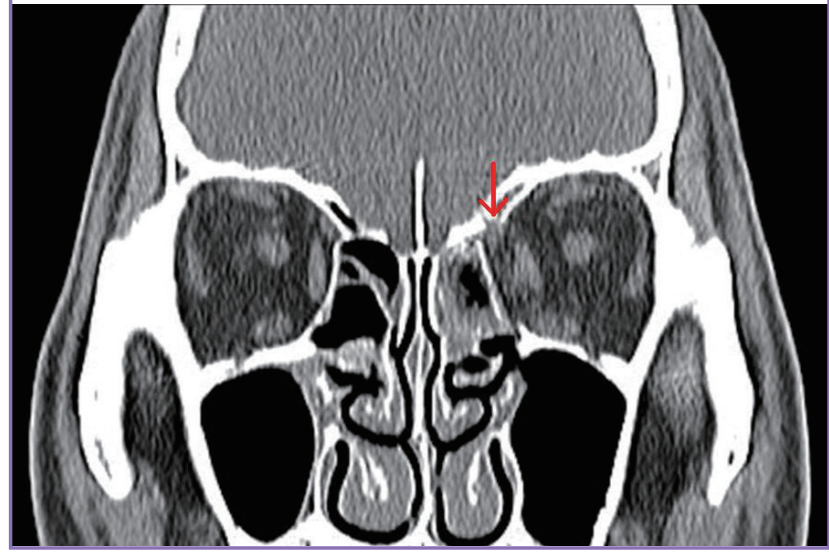

Fig. 8. A computed tomography scan after re-exploration

The Medpor implant was removed and the trapped muscle was released. A newly designed Medpor implant was inserted into the depressed orbital wall. After the surgery, a forced duction test showed no restriction of ocular motility (case 2).

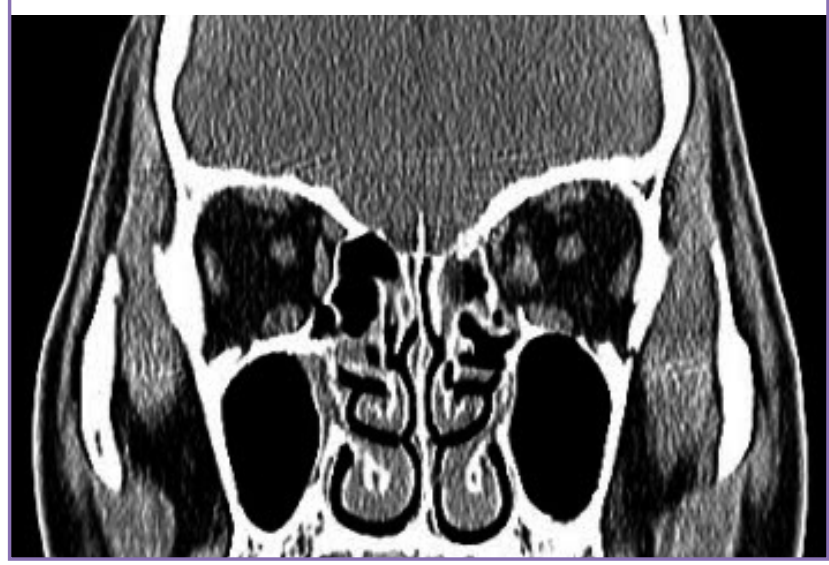

After the operation, he complained of aggravated diplopia and limited ocular motility. Limited elevation during adduction of the left eye was also observed. A postoperative CT scan showed that the superior oblique muscle was compressed by the swelling of adjacent soft tissue and hematoma. Acquired simulated Brown syndrome was strongly suspected. After two months, the patient's diplopia and limited ocular motility had improved and the patient was satisfied with his condition.

\section{DISCUSSION}

In 1950, Brown [6] reported a superior oblique tendon sheath syndrome characterized by limited elevation during adduction. He later redefined this sheath syndrome, dividing it into true
Table 1. Clinical features of Brown syndrome

Consistent features of Brown syndrome

Deficient or absent elevation in adduction

Normal or minimally deficient elevation in abduction

Mild elevation deficit in primary position

Positive forced ductions up and in

Minimal or no superior oblique overaction

Variable features of Brown syndrome

Down-shoot in adduction

Divergence in the up gaze causing a V-pattern (Y subtype)

Widening of the palpebral fissure during adduction

Anomalous head posture (chin up and contralateral face turn)

Hypotropia in primary position

sheath syndrome and simulated sheath syndrome [2]. Although the clinical features of true and simulated sheath syndrome are similar, true sheath syndrome is always congenital and persistent, and does not show spontaneous recovery. In 1975, Parks and Brown [1] reviewed 24 cases of true sheath syndrome, but did not find a tight or heavy anterior sheath surrounding the superior oblique tendon. The name was then changed to Brown syndrome and the reference to the anterior sheath was dropped [7].

Brown syndrome can be classified as congenital or acquired based on its cause. The cause of congenital Brown syndrome is an anomaly of the trochlear and/or superior oblique tendon, whereas acquired Brown syndrome can be caused by infection, inflammatory disease, trauma, and periocular or sinus surgery [3]. The clinical features of Brown syndrome include limited elevation during adduction, severe limitations in a forced duction examination, lengthening of the palpebral fissure, divergence in the upward gaze manifesting as a V-pattern, and hypotropia in primary position (Table 1) [8]. The differential diagnosis for restricted elevation during adduction includes pseudo-Brown syndrome, which is caused by paresis of the inferior oblique muscle. Pseudo-Brown syndrome is characterized by a negative forced duction examination, an A-pattern in the upward gaze, ipsilateral superior oblique overreaction, and intorsion in the primary gaze that increases in the upward gaze [9].

Some cases of acquired simulated Brown syndrome after orbital wall fracture operations have been reported. Lauer et al. [10] reported a case of Brown syndrome diagnosed following the repair of an orbital roof fracture. Hwang and Lim [5] described a case of Brown syndrome after the reconstruction of a medial wall fracture and observed that the tendon and muscle fiber near the trochlea were hypertrophied. Seo et al. [4] reported a case of Brown syndrome after a medial wall fracture reconstruction caused by the superior oblique muscle process between an implant and the posterior border of the fracture. Adulkar et 
al. [11] likewise described a case of Brown syndrome caused by the superior oblique process in a medial wall trapdoor fracture.

Due to the anatomical location of the superior oblique muscle, surgical procedures treating medial blowout fractures are considered a potential cause of acquired simulated Brown syndrome. In addition, direct or indirect trauma of the superior oblique muscle, edema, inflammation, and hemorrhage may be involved in the pathogenesis of acquired simulated Brown syndrome [5]. In the three cases reported here, the patients had impaired ocular motility involving limited elevation during adduction as well as positive forced duction examinations. When orbital CT scans were performed, we observed that the superior oblique muscle was trapped between the Medpor implant and the fracture margin. This condition is sometimes difficult to differentiate from inferior oblique paresis, but can be confirmed with a positive forced duction test. Once muscle entrapment is confirmed, prompt surgical release is essential because the muscle may later be fibrosed by cicatricial adhesions.

The surgical reconstruction of a blowout fracture involves a risk of external ocular muscle entrapment [12]. Therefore, it is essential to determine whether the external ocular muscle is trapped between the inserted implant and the fracture margin. Superior oblique muscle entrapment is uncommon and is therefore seldom considered a possibility during the identification of the superior margin of the medial orbital wall. The surgeon should always consider the possibility of Brown syndrome if an implant is put in place without clearly identifying the fracture margin, and also should perform a forced duction test after inserting the implant in order to ensure that all extraocular muscles were released.

\section{REFERENCES}

1. Parks MM, Brown M. Superior oblique tendon sheath syn- drome of Brown. Am J Ophthalmol 1975;79:82-6.

2. Brown HW. True and simulated superior oblique tendon sheath syndromes. Doc Ophthalmol 1973;34:123-36.

3. Park BC, Kim YH, Kim TG, et al. Treatment of posttraumatic facial deformity patient with Brown's syndrome: case report. J Korean Cleft Palate-Craniofac Assoc 2010;11:33-6.

4. Seo IH, Rhim JW, Suh YW, et al. A case of acquired Brown syndrome after surgical repair of a medial orbital wall fracture. Korean J Ophthalmol 2010;24:53-6.

5. Hwang JU, Lim HT. Acquired simulated brown syndrome following surgical repair of medial orbital wall fracture. Korean J Ophthalmol 2005;19:80-3.

6. Brown HW. Congenital structural anomalies of the muscle. In: Allen JH, editor. Symposium on strabismus: trans new orleans acad ophthalmol. St. Louis: CV Mosby; 1950. p.20536.

7. Manley DR, Alvi RA. Brown's syndrome. Curr Opin Ophthalmol 2011;22:432-40.

8. Von Noorden GK. Binocular vision and ocular motility: theory and management of strabismus. 3rd ed. St. Louis: Mosby; 1996.

9. Bae EH, Kim SJ. Typical and atypical Brown's syndrome. J Korean Ophth Soc 1987;28:1357-63.

10. Lauer SA, Sauer H, Pak SM. Brown's syndrome diagnosed following repair of an orbital roof fracture: a case report. J Craniomaxillofac Trauma 1998;4:20-2.

11. Adulkar N, Kim U, Shetty S. Superior oblique muscle entrapment in orbital fracture presenting as acquired brownlike syndrome: a case report and review of literature. Ophthal Plast Reconstr Surg 2014;30:e26-8.

12. Lee JH, Lee YJ, Kim SM, et al. A rare case of postoperative traumatic optic neuropathy in orbital floor fracture. Arch Plast Surg 2014;41:432-4. 